\title{
Jet-disk connection in OJ287
}

\author{
Mauri J. Valtonen ${ }^{1,2}$, Tuomas Savolainen ${ }^{3}$ and Kaj Wiik ${ }^{2}$ \\ ${ }^{1}$ Helsinki Institute of Physics, FIN-00014 University of Helsinki, Finland \\ email: mvaltonen2001@yahoo.com \\ ${ }^{2}$ Dept. of Physics \& Astronomy, Tuorla Observatory, University of Turku, 21500 Piikkiö, \\ Finland \\ email: kaj.wiik@utu.fi \\ ${ }^{3}$ Max-Planck-Institut für Radioastronomie, Auf dem Hügel 69, D-53121 Bonn, Germany \\ email: tsavolainen@mpifr-bonn.mpg.de
}

\begin{abstract}
A model for OJ 287 consisting of two orbiting black holes has been constructed using optical light curve data. The model has successfully predicted the occurrence of sharp optical outbursts of OJ 287 for the past 15 years. Here we test if also the variations in the radio jet position angle can be explained within the framework of this same model, which has most of its parameters fixed by the timing of the optical flares. The model applied here has only three free parameters left, the (trivial) zero point of the jet position angle, the time lag between changes in the disk and jet orientations, and the zero point of the viewing angle. Despite its simplicity and the small number of free parameters, the model appears to be able to reproduce the main properties of the observed position angle variations during the past 30 years. The best fits are obtained when the time lag is either $\sim 4$ or $\sim 14$ years. However, the jet orientation seems to be unrelated to the direction of the spin of the primary black hole. This implies, assuming that the basic model is correct, that the mean orientation of the jet is determined by the orientation of the inner accretion disk, not by the spin axis of the black hole.
\end{abstract}

Keywords. quasars, jets, binary black holes

\section{Introduction}

Many quasars and other extragalactic radio sources possess parsec scale jets where the jet direction varies with time - often wobblingly. There are several possible explanations for this behaviour, including rotation of the flow, propagation of current-driven or Kelvin-Helmholtz instabilities, and precession (and/or nodding motion) of the black hole's accretion disk due to a periodic perturbation by a possible binary companion.

BL Lac object OJ 287 is one of the best known candidates for harbouring a supermassive binary black hole $(\mathrm{BBH})$. In the case of OJ 287 the primary evidence for a $\mathrm{BBH}$ comes from the optical light curve which shows periods of outbursts at regular 12 year intervals (Sillanpää et al. 1988). The binary model for OJ 287 has predicted the optical variations in OJ 287 from 1995 until 2030 (Lehto \& Valtonen 1996, Sundelius et al. 1997). For the first fifteen years the brightness variations have followed the model with great accuracy (Valtonen et al. 2011). The flares related to the two sharp impacts in this period were predicted correctly within a few days. The first sharp flare was expected to begin on Nov 3, 1995 (as reported in a meeting in Oxford six weeks earlier) and the prediction was correct to the day (Sundelius et al. 1996), while the second one was expected to begin on Sept 10, 2007 and the prediction was an equal success (Valtonen \& Lehto 1997, Valtonen et al. 2008). The ability to predict the optical light curve so accurately presents a great challenge to any alternative model.

Valtonen et al. (2006) showed that at least three different mechanisms have to be included in a satisfactory BBH model for OJ 287: impacts of the secondary BH on the 
primary disk, tidal influence on the accretion flow, and the jet wobble. The first mechanism is responsible for the double peak structure of the sharp outbursts, the second one causes flares of longer duration, while the third one is responsible for the $60 \mathrm{yr}$ cycle, with a $12 \mathrm{yr}$ modulation. It is the latter mechanism which is of interest here since it also changes the jet orientation angle in the sky.

\section{VLBI data}

We have collected published VLBI data from the literature with observations dating back to the early 1980s (Roberts et al. 1987, Gabuzda et al. 1989, Gabuzda \& Cawthorne 1996, Vicente et al. 1996, Fey et al. 1996, Tateyama et al. 1996, 1999, Fey \& Charlot 1997, Rantakyrö et al. 1998, Gabuzda \& Gomez 2001, Tateyama \& Kingham 2004, Ojha et al. 2004, Jorstad et al. 2001, 2005, Piner et al. 2007, D'Arcangelo et al. 2009). In addition, we have analysed 79 epochs of $15 \mathrm{GHz}$ VLBA observations from the MOJAVE database (Lister et al. 2009a), resulting in altogether 196 epochs of VLBI observations. These data were used to build a record of variations in the position angle (PA) of the parsec scale jet over a 30-year period.

The data were divided in two categories: $2-6 \mathrm{~cm}$ observations and $0.3-1.3 \mathrm{~cm}$ observations. The division has practical significance since the angular resolution and also the core position depend on the observing frequency (Blandford \& Königl 1979). At higher frequencies the knots emerging in the jet are seen earlier than at low frequencies. Because of the longer time coverage of the low frequency observations, we use them in the following discussion. There is also little deviation in the PA between different wavelengths in the 2-6 cm data, which justifies stacking these observations together.

We have not tried to back-extrapolate the ejection epochs of the knots since it easily leads to ambiguous results for inhomogeneous data sets with significant time gaps. Instead, at each epoch, we measured the mean position angle of the jet within the first milliarcsecond from the core and then took a yearly average of these values. Lister et al. (2009b) report angular speeds of $0.5-0.8 \mathrm{mas} \mathrm{yr}^{-1}$ for the components near the core in OJ 287, which means that we measure changes in the PA with a delay of at least $\sim 1-2 \mathrm{yr}$. We note here that our approach does not take into account any asymmetry in the brightness distribution across the jet, which may introduce additional uncertainty about the mean jet direction.

\section{Disk model}

The accretion disk of the primary $\mathrm{BH}$ is modelled by discrete non-interacting particles, 37200 in number. They are placed in circular coplanar orbits between 8 and 20 Schwarzschild radii of the primary black hole. This is the region which is mostly influenced by the gravitational effects of the secondary black hole. The dynamical model is described in detail in Valtonen et al. (2010). Note that all the parameters of the binary orbit in this model are fixed by the timing of the optical outbursts. We calculate the orbital elements of the disk particles at each time step, and take their average values for every calendar year. Then a sliding average over 10 years is calculated for every annual point. This smoothing process is necessary since the sound crossing time of the ring is of the order of ten years, and the smoothing does not happen automatically in our simulation, unlike in real gaseous disks. We note that this model is clearly oversimplified as it neglects viscosity and pressure in the disk, but we use it here to give a first approximation of the disk bending. A more physical model including viscosity is currently being worked on. 

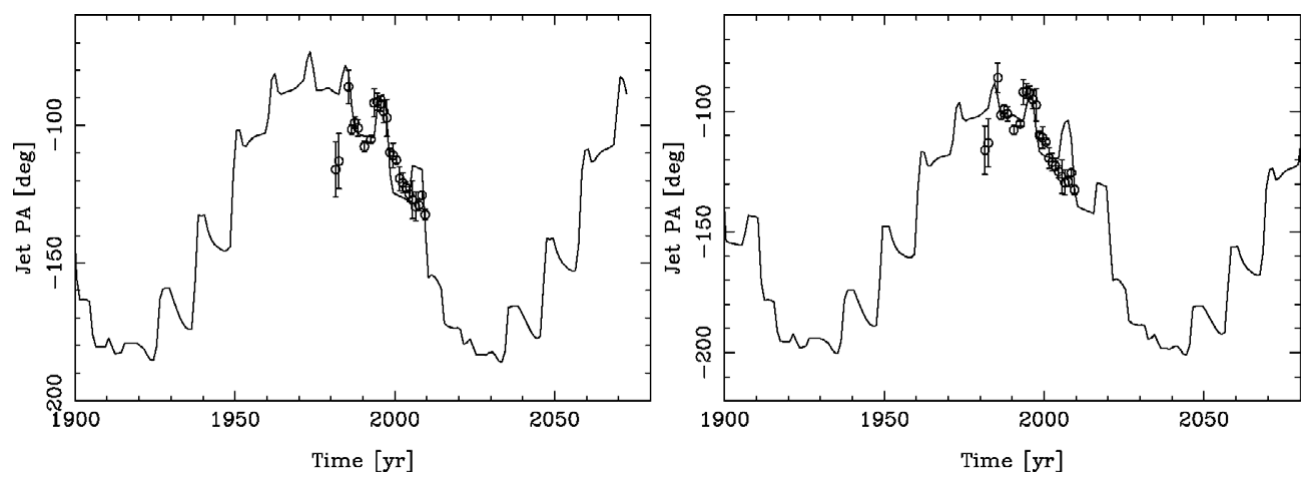

Figure 1. The line shows the variation of the jet angle as a function of time, based on the binary model of OJ 287. The viewing angle of the jet varies between 1 and 2 degrees. The circles represent the yearly average PA observed in the $2-6 \mathrm{~cm}$ wavelength range. The error bars represent the standard deviation of the PA when more than two observations per year were available. Left: A 4-year lag is introduced between changes in the accretion disk orientation and jet reorientation. Right: The same as the left-hand side, but the lag is 14 years.

The basic cycle of the disk precession due to perturbations of the secondary BH appears to be 120 yr. We may now assume that the jet lies along the rotation axis of the disk and when the disk orientation changes, the jet axis also changes, with some time lag. Figure 1 shows the predicted PA changes (solid line). Note that we have only three free parameters since everything else has been fixed by the optical model. The first parameter is the zero point of the radio position angle. It can be changed by sliding the theoretical curve up and down vertically in Figure 1. The second parameter is related to the time lag, and it is changed by sliding the theoretical curve horizontally along the time axis. The third parameter is the zero point of viewing angle, varying of which stretches or compresses the vertical scale. This last parameter can be constrained by requiring that the model is consistent with the viewing angles derived from long-term flux density variations.

Figure 1 shows the two best fits. Both have the same viewing angle range of 1-2 degrees, which is consistent with radio variability studies (e.g., Savolainen et al. 2010), but different values of time lags. The model with a 4 -yr delay between the changes in the accretion disk orientation and the re-orientation of the pc scale jet fits the data surprisingly well, except for the two earliest data points which are from four-station observations with the US VLBI Network. The poor $(u, v)$-coverage of these observations leaves the jet orientation more uncertain than in the rest of the data.

The model predicts that the jet PA turns clockwise by $\sim 50$ degrees during the next 10-20 years, with the exact timing depending on the chosen time lag. Interestingly, Agudo et al. (2010) reported that a new jet component recently appeared at PA $=200$ degrees in the $43 \mathrm{GHz}$ VLBA data. However, during the last few years there have been multiple ejections with widely differing position angles in the $43 \mathrm{GHz}$ jet (D'Arcangelo et al. 2009, Agudo et al. 2010), which may indicate that these components are moving kinks in the jet and therefore their apparent path may deviate from the mean jet direction. It will be important to follow the jet PA with $15 \mathrm{GHz}$ VLBI observations during the next few years in order to see if the mean direction of the jet continues to turn as predicted.

\section{Discussion}

As shown in Figure 1, the direction of the parsec scale radio jet of OJ 287 shows significant evolution on the plane of the sky. We have tested if it is possible to fit this 
observed evolution with a very specific binary black hole model developed for explaining the periodic optical outbursts of OJ 287. The answer appears to be yes. However, it will be important to check this result by using a more physical, hydrodynamical model of the accretion disk.

The binary motion affects both the accretion disk around the primary black hole and the spin of the primary black hole. Both effects are calculable in the binary model. The wobble of the accretion disk axis seems to be connected to the jet position angle in the sky and the changes are most likely transmitted by magnetic fields. On the other hand, the variations in the black hole spin direction are too slow to show up in the time scale that we are able to study. We may thus say that assuming our BBH model is correct, the jet direction is determined by the accretion disk, not by the spin axis of the black hole.

It has been previously noticed that the optical outbursts lag behind tidal influences in the disk by about three months (Valtonen et al. 2006). Considering the distance of the perturbed ring from the central black hole, the speed of transmission of particles from the disk to the jet would be of the order of the orbital speed of the disk. Thus it appears that in this model particles travel freely along magnetic field lines from the perturbed disk to the jet. The tilting of the accretion disk in the perturbed region $(8-20$ Schwarzschild radii from the central black hole) causes also the tilting of the radio jet.

\section{References}

Agudo, I., et al. 2010, in "Fermi meets Jansky - AGN in radio and gamma-rays", Savolainen, T., Ros, E., Porcas, R. W., \& Zensus, J. A., eds., 143

Blandford \& Königl 1979, ApJ, 232, 34

D'arcangelo, F. D., et al. 2009, ApJ, 697, 985

Fey, A. L., Clegg, A. W., \& Fomalont, E. B. 1996, ApJS, 105, 299

Fey, A. L. \& Charlot, P. 1997, ApJS 111, 95

Gabuzda, D. C., Wardle, J. F. C., \& Roberts, D. H. 1989, ApJL, 336, L59

Gabuzda, D. C. \& Cawthorne, T. V. 1996, MNRAS, 283, 759

Gabuzda, D. C. \& Gomez, J. L. 2001, MNRAS, 320, 49

Jorstad, S. G., et al. 2001, ApJS, 134, 181

Jorstad, S. G., et al. 2005, AJ, 130, 1418

Lehto, H. J., \& Valtonen, M. J. 1996, ApJ, 460, 207

Lister, M. L., et al. 2009a, AJ, 137, 3718

Lister, M. L., et al. 2009b, AJ, 138, 1874

Ojha, R. et al. 2004, ApJS, 150, 187

Piner, G., et al. 2007, AJ, 133, 2357

Rantakyrö, F., et al. 1998, A\&AS, 131, 451

Roberts, D. H., Gabuzda, D. C., \& Wardle, J. F. C. 1987, ApJ, 323, 536

Savolainen, T., et al. 2010, A\& A, 512, A24

Sillanpää, A., et al. 1988, ApJ, 325, 628

Sundelius, B., et al. 1996, ASP Conf.Ser. 110, 99

Sundelius, B., et al. 1997, ApJ, 484, 180

Tateyama, C. E., et al. 1996, PASJ, 48, 37

Tateyama, C. E. \& Kingham, K. A. 2004, ApJ, 608, 149

Tateyama, C. E., et al. 1999, ApJ, 520, 627

Valtonen, M. J. \& Lehto, H. J. 1997, ApJL, 481, L5

Valtonen, M. J., et al. 2006, ApJ, 646, 36

Valtonen, M. J., et al. 2008, Nature, 452, 851

Valtonen, M. J., et al. 2010, ApJ, 709, 725

Valtonen, M. J., et al. 2011, ApJ, in press

Vicente, L., Charlot, P., \& Sol, H. 1996, A\& A, 312, 727 


\section{Discussion}

EMmanoulopoulos: Is the model that you suggest stable from a MHD point of view?

VALTONEn: We have not carried out MHD simulations. However, the particle disk that we use is stable over 10000 years.

DE Gouveia Dal Pino: Is there any further evidence of SMBH binary systems?

VAltonen: OJ287 is the only one that came up in the Tuorla monitoring program of about 50 sources. But there are reasons to expect, based on cosmological models, that more will be discovered if the monitoring is extended to larger samples, say 1000. In addition, there are many rotating radio jets which may indicate a binary system.

FEnDT: The binary BH is responsible for precession. May it also affect the path of the jet by gravitational interactions (binding)?

VALTONEN: In the binary model, the secondary goes close to the jet once in every period. It has been noted that the optical flux drops dramatically at those times for a few weeks. One explanation is, as you mentioned, the gravitational jet bending at those times. 